\title{
Publisher's Erratum: Spinal-generated movement disorders: a clinical review
}

Pichet Termsarasab $b^{1,2^{*}}$, Thananan Thammongkolchai ${ }^{3}$ and Steven J. Frucht ${ }^{1}$

\section{Erratum}

Following publication of the original article [1], it was noticed that the video files for this article (included as supplementary material) were not functioning correctly. Please see below for the corrected files:

\section{Additional files}

Additional file 1: Video segment 1 demonstrates the phenomenology of spinal segmental myoclonus, priopriospinal myoclonus (PSM) and stiff limb syndrome. See section on Video segments for more details.

Additional file 2: Video segment 2 demonstrates the rich phenomenology of orthostatic tremor (OT) in five patients. See section on Video segments for more details.

Additional file 3: Video segment 3 demonstrates the phenomenology of painful legs-moving toes (PLMT) and painful hands-moving fingers (PHMF) syndromes. See section on Video segments for more details.

\section{Author details}

Department of Neurology, Movement Disorder Division, Icahn School of Medicine at Mount Sinai, New York, USA. ²Department of Medicine, Neurology Division, Faculty of Medicine, Siriraj Hospital, Mahidol University, Bangkok, Thailand. ${ }^{3}$ Department of Neurology, University Hospitals Case Medical Center, Cleveland, USA.

Received: 26 August 2016 Accepted: 26 August 2016

Published online: 22 November 2016

\section{Reference}

1. Termsarasab P, et al. Spinal-generated movement disorders: a clinical review. J Clin Mov Disord. 2015;2:18.

\footnotetext{
* Correspondence: pichetterm@gmail.com

${ }^{1}$ Department of Neurology, Movement Disorder Division, Icahn School of Medicine at Mount Sinai, New York, USA

2Department of Medicine, Neurology Division, Faculty of Medicine, Siriraj Hospital, Mahidol University, Bangkok, Thailand
} 